\title{
Research on the Relationship Between Psychological Control Source and Self-Defense Mechanism of Young Teachers in Universities and Colleges
}

\author{
Shoudong TANG* \\ Heihe University, Heihe City, Heilongjiang, China \\ Email: 1109720344@qq.com.
}

Keywords: college young teachers, psychological control source, self-defense mechanism

Abstract. This research adopts the questionnaire of young university teachers psychological control source and its present situation and the characteristics of defense mechanism has carried on the detailed analysis and discussion, and further discusses the young university teachers psychological control source and self-defense mechanism, drawing the following conclusions: (1) intenality type has a significant negative correlation with immature defense mechanisms and a significant positive correlation with mature defense mechanisms.(2) the psychological control source of young teachers in colleges and universities has a significant predictive effect on their self-defense mechanism.

Psychological control source is a relatively stable psychological trait. Different types of psychological control source have different performance in behavioral response, attributions of success or failure and attitudes. The external behavior is the application of self-defense mechanism. The purpose of the self-defense mechanism is to reduce the pain and maintain the inner balance, that is, to cope with the self-driven, the pressure of the superego and the requirements of the external reality, so as to reduce and relieve the psychological tension and obtain the psychological measures and defense means of the inner balance. The rest of the mind thinks that the controller has an internal connection.

\section{Research Technique}

With Internality, Powerful Others and Chance Scale(IPC) and Defense Style Questionnaire(DSQ), 247 subjects were randomly selected from a number of in-service teachers of undergraduate colleges and universities in Heilongjiang Province. 235 effective questionnaires were collected and screened, with an effective rate of $95.14 \%$. All data were entered into the computer, and SPSS17.0 for Windows statistical software was used for statistical processing.

\section{Result and Analysis}

The correlation analysis of psychological control source and self-defense mechanism of young teachers in universities and colleges. The correlation between the psychological control source and the self-defense mechanism of young teachers in universities and colleges was investigated by the statistical method of Person product and difference correlation. The results (table 2-1) showed that the correlation between the factors of the psychological control source and the factors of the defense mechanism reached a significant level.

Immature defense mechanism is negatively correlated with internality, positively correlated with powerful others and opportunities.Mature defense mechanism is positively correlated with internality, powerful others and opportunities.Intermediate defense mechanism is positively correlated with powerful others and opportunities, negatively correlated with internality, but not significantly. 
Table 2-1 correlation between psychological control source and self-defense mechanism and various factors of young teachers in universities and colleges ( $\mathrm{r}$ value)

\begin{tabular}{cccc}
\hline & internality & powerful others & chances \\
\hline immature defense mechanism & $-0.146^{*}$ & $0.451^{* *}$ & $0.531^{* *}$ \\
$\begin{array}{c}\text { mature defense mechanism } \\
\text { intermediate defense } \\
\text { mechanism } \\
\text { conceal factor }\end{array}$ & $0.177^{* *}$ & $0.277^{* *}$ & $0.256^{* *}$ \\
\hline
\end{tabular}

note: *means $\mathrm{p}<0.05, * *$ means $\mathrm{p}<0.01$

Regression analysis of the psychological control source of the young college teachers' self-defense mechanism.For the analysis of young university teachers psychological control the relationship between the source and self-defense mechanism of each factor, this study to mental control source scale of internality, the opportunities, the powerful others 3 factors as independent variables, respectively, in the defense mechanism questionnaire mature defense mechanism, immature defense mechanisms and type of defense mechanism among three factors as the dependent variable, using the method of stepwise regression multivariate linear regression analysis, the results showed that (table 2-2) :chance, powerful others and internality all entered the regression equation and reached the significance level, indicating that the psychological control source has a significant predictive effect on the three types of defense mechanisms, indicating that it is reliable to use the psychological control source to predict the specific performance of the three defense mechanisms.

Table 2-2 regression of the self-defense mechanism of young college teachers to the source of psychological control

\begin{tabular}{ccccccc}
\hline \multirow{2}{*}{ dependent variable } & $\begin{array}{c}\text { independent } \\
\text { variable }\end{array}$ & $B$ & $t$ & $F$ & $R^{2}$ & constant \\
& chance & 0.596 & $5.644^{* * * *}$ & & & \\
immature & powerful others & 0.298 & $2.778^{* *}$ & $32.783^{* * *}$ & 0.341 & 3.143 \\
& internality & -0.331 & $-3.050^{* *}$ & & & \\
& powerful others & 0.295 & $2.36^{* *}$ & & & \\
mature & chance & 0.283 & $2.31^{*}$ & $8.228^{* * *}$ & 0.105 & 3.380 \\
& powerful others & 0.279 & $2.26^{* *}$ & & & \\
central type & powerful others & 0.331 & $3.831^{* * *}$ & $33.292^{* *}$ & 0.258 & 3.050 \\
& chance & 0.290 & $3.418^{* * *}$ & & & \\
\hline
\end{tabular}

note: *means $\mathrm{p}<0.05$, **note: $\mathrm{p}<0.01$, ***means $\mathrm{p}<0.001$

\section{Discussion}

There is a significant correlation between the psychological control source and the self-defense mechanism. Through the progressive analysis of the correlation matrix between the psychological control source and the self-defense mechanism, it can be seen that there is also a significant correlation between the factors of the psychological control source and the factors of the self-defense mechanism. Internality has a significant negative correlation with immature defense mechanism, a significant positive correlation with mature defense mechanism, and no correlation 
with intermediate defense mechanism. It indicates that the higher the score of internality, the higher the frequency of using the mature defense mechanism to solve problems; on the contrary, the lower the score of internality, the higher the frequency of using the immature defense mechanism.chance and powerful others have significant positive correlation with the three defense mechanisms.It can be seen that there is a complex relationship between the psychological control source and the self-defense mechanism of young teachers in colleges and universities.

The psychological control source of young teachers in colleges and universities can effectively predict the performance of the self-defense mechanism. Three factors of psychological control source have significant predictive effect on immature defense mechanism.Specific forecast, the internality's control source of young university teachers is negative to predict immature defense mechanisms and opportunities and powerful control source is positively predict immature defense mechanisms, which consistent with the correlation analysis, show that the main sources of young university teachers psychological control intenality, the lower internality is, the opportunities and the higher the powerful others individual, the easier it is to adopt immature defense mechanisms.

The three factors of psychological control source help are competing to predict the performance of mature defense mechanism, which is consistent with the correlation analysis, indicating that the psychological control source of young teachers in colleges and universities is internality. The higher the internality, the more likely the individual is to adopt mature self-defense mechanism.

Chance and powerful other factors in psychological control sources have significant positive predictive effects on intermediate defense mechanism. As the source of young university teachers psychological control part of the intenality and middle type self-defense mechanism factor positively correlated, and negatively correlated with another part of the factors, which lead to intenality and has no impact on intermediate defense mechanism on the whole, and intenality has not entered the forecast, so comparative psychological control source type of immature defense mechanism of prediction, the intenality in certain circumstances, when the young university teachers psychological control source opportunities and powerful people, the higher the individuals among the more inclined to adopt self defense mechanism, the second type will tend to adopt immature defense mechanisms, This is consistent with the application characteristics of the self-defense mechanism of young teachers in universities and colleges, which fully shows that the prediction of the self-defense mechanism by the psychological control source of young teachers in universities and colleges is reliable.

Although the influence of others is an external control factor, it is a human factor. Individuals have a certain initiative on them and can influence them in some special ways, so as to control the development and results of things to a certain extent. As at the beginning of the career development of young university teachers, because of its professional foundation still owe thick, teaching style is not fully mature, scientific background slightly weak, lack of professional feeling of competence to a certain extent, in the face of the knowledge structure of the relatively static and dynamic social development there are some contradictions between the needs and expectations of their own teaching effectiveness is difficult to make effective judgment, especially in the scientific research ability is to ascend, they need to be expert teachers to guide and help, so in the forecast of three kinds of defense mechanisms have emerged in the powerful factors of others is reasonable.

The reason for the entry of the chance factor is simple, although it is uncontrollable external factors, but everyone wants to be able to get his favor.Type from the regression coefficient opportunities for immature defense mechanism of prediction effect is best, followed by the type of defense mechanism among, the last is mature defense mechanism, which fully shows the young teachers in colleges and universities is a mature community, the more inclined to chance, the more easy to adopt immature defense mechanisms, more inclined to intenality sex is more easy to adopt mature defense mechanisms. 


\section{Conclusion}

There is also a significant positive correlation between powerful others and the three defense mechanisms. All the factors were significantly correlated with powerful others, except that depression and communication tendency were not correlated with powerful others.External control is positively correlated with all defense mechanisms and factors. Young teachers in colleges and universities are a mature group. The more inclined they are to intenality, the more likely they are to adopt mature defense mechanism; the more inclined they are to chance, the more likely they are to adopt immature defense mechanism. The psychological control source of young teachers in colleges and universities has a significant predictive effect on their self-defense mechanism.

\section{References}

[1] Shoudong Tang,Yu Ding,Hong Sun.Study on psychological defense mechanism of young teachers in universities and colleges[J].Industry and technology formula,2015,14(12):110-111.

[2] Shoudong Tang, Di Wang, Yanmin Li.Study on the source of occupational psychological control of young teachers in newly-established undergraduate universities[J].Science and education materials(late),2015(01):18-19+26.

[3] Alu Wu.Research on psychological defense mechanism and mental health of public security college students[J].Journal of Guizhou police officer vocational college,2019,31(02):118-122+129.

[4] Qiuyun Wang,Naiyi Wang.A follow-up study on psychological resilience and defense mechanism in high school students[J]. Mental health education in primary and secondary schools, 2018(21):9-11+14.

[5]Shu Chen,Juan Ma,Jinfu Zhu.Occupational burnout and psychological control source of teachers in higher vocational colleges 、 coping style[J].Chinese journal of mental health,2014,28(04):293-297. 